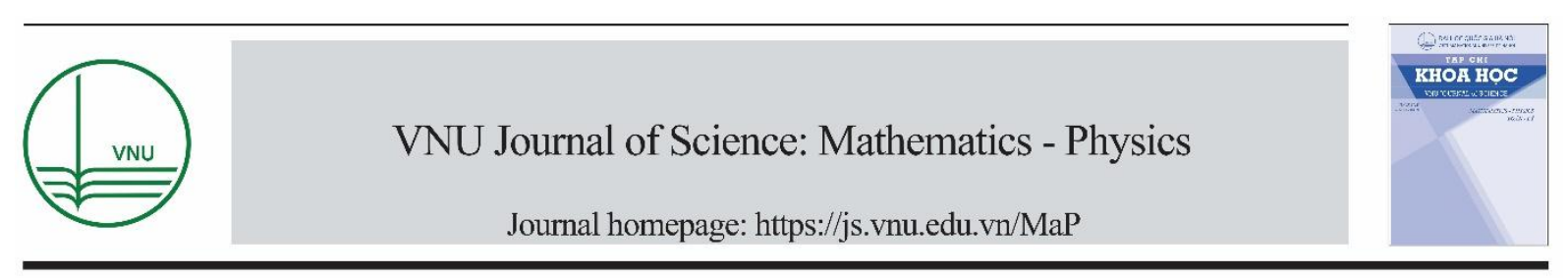

Original Article

\title{
Prediction of Weinberg Angle in Discretized Kaluza-Klein Theory
}

\author{
Nguyen Van Dat* \\ Faculty of Physics, VNU University of Science, 334 Nguyen Trai, Hanoi, Vietnam
}

Received 09 May 2019

Accepted 28 May 2019

\begin{abstract}
In the discretized Kaluza-Klein theory (DKKT), the gauge fields emerge as components of gravity with a single coupling constant. Therefore, it provides a new approach to fix the parameters of the Standard Model, and in particular the Weinberg angle. The study results show that in the approach using DKKT, the predicted value of Weinberg angle is exactly the one measured in the electron-positron collider experiment at $\mathrm{Q}=91.2 \mathrm{GeV} / \mathrm{c}$. The result is compared to the one predicted by the group theoretic methods.

Keywords: Weinberg angle, discretized Kaluza-Klein theory, DKKT.
\end{abstract}

\section{Introduction}

The Weinberg angel $\theta_{W}$ [1-3] is the most important parameter of the Standard model, which relates the two coupling constant $\mathrm{g}$ and g' corresponding to the two underlying gauge groups $S U(2)_{L}$ and $U(1)_{Y}$. It can be introduced by the following mixing of the gauge field $W_{\mu}^{3}$ and $B_{\mu}$ to form the physical photon and $Z^{0}$ fields

$$
\left[\begin{array}{c}
A_{\mu} \\
Z_{\mu}^{0}
\end{array}\right]=\left[\begin{array}{cc}
\cos \theta_{W} & \sin \theta_{W} \\
-\sin \theta_{W} & \cos \theta_{W}
\end{array}\right]\left[\begin{array}{c}
B_{\mu} \\
W_{\mu}^{3}
\end{array}\right]
$$

In terms of the coupling constants g and g', the Weinberg angle can be expressed as

\footnotetext{
${ }^{*}$ Corresponding author.

Email address: dnvdat@gmail.com
}

https//doi.org/ 10.25073/2588-1124/vnumap.4350 


$$
\begin{aligned}
& \cos \theta_{W}=\frac{g}{\sqrt{g^{2}+g^{\prime 2}}} \\
& \sin \theta_{W}=\frac{g^{\prime}}{\sqrt{g^{2}+g^{\prime 2}}}
\end{aligned}
$$

Therefore, it relates the masses of the $W^{ \pm}$and $Z^{0}$ as follows

$$
m_{W}=m_{Z} \cos \theta_{W}
$$

It can also relates the coupling constants $g$ and g' to the electric charge

$$
e=g \sin \theta_{W}=g^{\prime} \cos \theta_{W}
$$

Originally, since the Weinberg angle is a free unfixed parameter of the Standard Model, its value can only be determined by experimental measurement. It's most precise measurements carried out in electron-positron collider experiments at a value of $\mathrm{Q}=91.2 \mathrm{GeV} / \mathrm{c}$, have given the value [4]

$$
\sin ^{2} \theta_{W}=0.231200 \pm 0.00015
$$

Due to the radiative corrections and renormalization effects, $\sin ^{2} \theta_{W}$ is a running constant, giving different values at different energies. For example, at $\mathrm{Q}=0.16 \mathrm{GeV} / \mathrm{c}$ the value of $\sin ^{2} \theta_{W}$ is $0.2397 \pm 0.0013$ and at $\mathrm{Q}=7-8 \mathrm{TeV} / \mathrm{c}$, it is 0.23142 .

The theoretical prediction of the Weinberg angle is possible in unified theories when only one coupling constant is used instead of $g$ and g'. In this paper, we will refer to the works of Fairlie $[5,6]$ as an example of such theories. Essentially, these works are based on the simple gauge group $S U(2 \mid 1)$ having only one coupling constant leading to the prediction of the Weinberg angle

$$
\sin \theta_{W}=0.25
$$

It is remarkable that the predicted value of the Weinberg angle is very far from the experimental value. It means that it is not at the measured electroweak scale energy, but at another one. At this energy scale the two gauge groups $S U(2)_{L}$ and $U(1)$ are embedded into a single simple one, which gives only one coupling constant. By choosing different simple unification gauge group like $S U(5)$, $E_{8}, \ldots$ we might have different predictions, but since their energy scales are far from the electroweak one, those cannot reproduce the above experimental value.

In this paper, we follow a different approach, using the discretized Kaluza-Klein theory developed by Viet and Wali [7-12] to predict the Weinberg angle. The new feature of this approach is that it is not a group theoretic but a geometric one. Surprisingly, this approach have given the value $\sin ^{2} \theta_{W}$ as 0.23077 , which is very close to the experimental value.

This paper organized as follows. In the section 2, we will briefly review the Discretized KaluzaKlein theory (DKKT). In the section 3, we will calculate the Weinberg angle in this framework. In the section 4, we will compare the obtained prediction with ones of Fairlie. In the section 5, we will discuss the results and physical implications. 


\section{Overview of discretized Kaluza-Klein theory}

In 1994, Landi, Viet and Wali [6] have revised the work of Felder, Frohlich and Chamseddine [13], which extend the non-commutative geometric model of Connes and Lott [14]. Surprisingly, the zero modes of the original Kaluza-Klein theory $[15,16]$ have emerged. This inspired Viet and Wali to develop the DKKT with a full physical content having a pair of gravity, vector and scalar fields. In each pair one field is massless and the other is massive. Based on these results, the NCG can be used to construct the theories in the space-time extended by discrete extra dimensions. The main advantage of DKKT is that it can avoid the infinite tower of massive fields in the usual Kaluza-Klein one with continuous extra dimensions. Discrete extra space-time means that one have different sheets of the usual space-time. For instance, if the extra dimension consists of two points, one must have two copies of the space-time.

In order to incorporate the weak and strong interactions into this theory, one must extend it to the case of nonabelian gauge vector fields. In 2015, Viet and Du have proven that in the case of the discrete extra dimension of two points it is possible to extend DKKT to include the nonabelian gauge fields in the two cases i) The gauge fields must be the same for both sheets of space-time or ii) The gauge field on at least one sheet must be abelian. Viet, Dat, Han and Wali, have applied this result to construct the Einstein-Yang-Mills-Dirac theories, having QCD coupled to gravity and electroweak couple to gravity theories as special cases. Viet has also proposed to extend DKKT to include two discrete extra dimensions, each having two points, leading to a unified theory where all four interactions and the Higgs fields emerge as components of a generalized gravity.

In this article we will focus on the case, when the electroweak interaction emerges as a component of gravity and couples to quarks and lepton as suggested in [12].

The construction is based on the spectral triplet. The first element of spectral triple is Hilbert space $\mathcal{H}=\mathcal{H}_{L} \oplus \mathcal{H}_{R}$. So, the generalization of spinor is direct sum of two chiral spinors,

$$
\Psi=\left[\begin{array}{l}
\psi_{L} \\
\psi_{R}
\end{array}\right] .
$$

The second element is the Algebra using for function operations $\mathcal{A}=\mathcal{A}_{L} \oplus \mathcal{A}_{R}$, where $\mathcal{A}_{I}=\mathcal{C}^{\infty}\left(\mathcal{M}^{4}\right), \mathrm{I}=\mathrm{L}, \mathrm{R}$. The elements of this algebra is represented by 0 -form diagonal matrix $\mathrm{F}$,

$$
F(x)=\left[\begin{array}{ll}
f_{L}(x) & 0 \\
0 & f_{R}(x)
\end{array}\right] .
$$

The third element is the Dirac operator, which that can be defined as an extension of the normal Dirac operator as $D=d . \mathbf{e}+\Theta$, where $d=\gamma^{\mu} \partial_{\mu}$ is usual Dirac operator in the four dimensions spacetime $\mathcal{M}^{4}$

$$
\begin{aligned}
& D=\left[\begin{array}{ll}
\gamma^{\mu} \partial_{\mu} & -i m \gamma^{5} \\
i m \gamma^{5} & \gamma^{\mu} \partial_{\mu}
\end{array}\right], \\
& \Theta=\left[\begin{array}{ll}
0 & -i m \gamma^{5} \\
i m \gamma^{5} & 0
\end{array}\right] .
\end{aligned}
$$

With NCG space-time defined with the above spectral triplet, we can calculate the derivative of the 0 -forms by acting the Dirac operator on function $\mathrm{F}$ as follows 


$$
\begin{gathered}
D F=[D, F] \\
=\left[\begin{array}{ll}
\gamma^{\mu} \partial_{\mu} f_{L}(x) & i m \gamma^{5}\left(f_{L}(x)-f_{R}(x)\right) \\
i m \gamma^{5}\left(f_{L}(x)-f_{R}(x)\right) & \gamma^{\mu} \partial_{\mu} f_{R}(x)
\end{array}\right]
\end{gathered}
$$

We can rewrite it in the following form

$$
\begin{aligned}
& D F=D X^{\mu}\left[D_{\mu}, F\right]+D X^{5} \sigma^{\dagger}\left[D_{5}, F\right] \\
& =D X^{\mu} \partial_{\mu} F+D X^{5} \sigma^{\dagger} \partial_{5} F
\end{aligned}
$$

where

$$
D_{5}=\left[\begin{array}{ll}
0 & -m \\
m & 0
\end{array}\right], \sigma^{\dagger}=\left[\begin{array}{ll}
0 & -1 \\
1 & 0
\end{array}\right] .
$$

If we use the representation of Dirac matrix, $D X^{\mu}$ can be replaced by the generalized $\gamma$-matrices

$$
\Gamma^{\mu}=\left[\begin{array}{ll}
\gamma^{\mu} & 0 \\
0 & \gamma^{\mu}
\end{array}\right], \Gamma^{5}=\left[\begin{array}{ll}
0 & i \gamma^{5} \\
-i \gamma^{5} & 0
\end{array}\right]
$$

Since the derivative of a 0 -form is a 1 -form, we can extend the module of 1 -forms, which is the generalization of the vector field in NCG to the following form

$$
\begin{aligned}
U & =\Gamma^{M} U_{M}=\Gamma^{\mu} U_{\mu}+\Gamma^{5} U_{5} \\
& =\left[\begin{array}{ll}
\gamma^{\mu} u_{\mu L} & i \gamma^{5} u_{5 R} \\
-i \gamma^{5} u_{5 L} & \gamma^{\mu} u_{\mu R}
\end{array}\right]
\end{aligned}
$$

where $U_{M}$ is generalized functions ( 0 -forms). The 1-form $U$ contains two vectors and two scalars.

Now we can define the 2-form to be used as generalized field strength or curvature. The 2-forms must extend from the derivatives of 1-forms. We have to define wedge product of two 1-forms as follows

$$
\begin{aligned}
D X^{\mu} & \wedge D X^{v}=-D X^{v} \wedge D X^{\mu} \\
D X^{\mu} & \wedge D X^{5}=-D X^{5} \wedge D X^{\mu} \\
D X^{5} & \wedge D X^{5} \neq 0, \\
(U \wedge V)_{\mu \nu} & =-(U \wedge V)_{v \mu}=\frac{1}{2}\left(U_{\mu} V_{v}-U_{v} V_{\mu}\right) \\
(U \wedge V)_{\mu 5} & =-(U \wedge V)_{5 \mu}=\frac{1}{2}\left(\tilde{U}_{\mu} V_{5}-U_{5} V_{\mu}\right) \\
(U \wedge V)_{55} & =\tilde{U}_{5} V_{5}
\end{aligned}
$$

where tidle operation " $\sim$ " on a generalized function is defined as follows

$$
\tilde{F}=f_{+} \mathbf{e}-f_{-} \mathbf{r}, f_{ \pm}=\frac{1}{2}\left(f_{1} \pm f_{2}\right)
$$

and 


$$
\mathbf{e}=\left[\begin{array}{ll}
1 & 0 \\
0 & 1
\end{array}\right], \mathbf{r}=\left[\begin{array}{ll}
1 & 0 \\
0 & -1
\end{array}\right] .
$$

Exterior derivative of 1 -forms is given as

$$
\begin{aligned}
& D U=[D, U]=D X^{M} \wedge D X^{N}(D U)_{M N} \\
& (D U)_{\mu \nu}=\frac{1}{2}\left(\partial_{\mu} U_{v}-\partial_{v} U_{\mu}\right)=-(D U)_{v \mu}, \\
& (D U)_{\mu 5}=\frac{1}{2}\left(\partial_{\mu} U_{5}-m\left(U_{\mu}-\tilde{U}_{\mu}\right)\right)=-(D U)_{5 \mu}, \\
& (D U)_{55}=m\left(U_{5 L}+U_{5 R}\right) .
\end{aligned}
$$

\section{The prediction of the Weinberg angle in DKKT}

The gauge field A in DKKT takes the following form

$$
\begin{aligned}
A & =\left(\begin{array}{cc}
\gamma^{\mu} a_{\mu L} & i \gamma^{5} a_{5}^{\dagger} \\
-i \gamma^{5} a_{5} & \gamma^{\mu} a_{\mu R}
\end{array}\right) \\
& =\Gamma^{\mu} A_{\mu}+\Gamma^{5} \sigma^{\dagger} A_{5} \\
& =D X^{\mu} A_{\mu}+D X^{5} \sigma^{\dagger} A_{5} .
\end{aligned}
$$

So, the gauge fields $a_{L}, a_{R}$ and complex scalar field $a_{5}$ are choosen as elements of the following $2 \times 2$ matrices

$$
\begin{aligned}
A_{\mu} & =\left(\begin{array}{ll}
a_{L \mu} & 0 \\
0 & a_{R \mu}
\end{array}\right), \\
A_{5} & =\left(\begin{array}{ll}
a_{5} & 0 \\
0 & a_{5}^{\dagger}
\end{array}\right) .
\end{aligned}
$$

Now we can introduce the physical fields $W_{\mu}^{a}(x)$ and $B_{\mu}(x)$ by assigning

$$
\begin{aligned}
& a_{L \mu}=g W_{\mu}^{a}(x) \frac{\tau^{a}}{2} \otimes 1_{4} \otimes 1_{N_{F}}-g^{\prime} \frac{Y_{L}}{2} B_{\mu}(x) \otimes 1_{2} \otimes 1_{N_{F}} \\
& a_{R \mu}=-g^{\prime} \frac{Y_{R}}{2} B_{\mu}(x) \otimes 1_{N_{F}} \\
& a_{5}=f_{k}\left[\begin{array}{ll}
h_{0}-\frac{m}{f_{k}} & h_{1}^{*} \\
-h_{1} & h_{0}^{*}-\frac{m}{f_{k}}
\end{array}\right], H=\left[\begin{array}{l}
h_{0} \\
h_{1}
\end{array}\right] .
\end{aligned}
$$

where $\mathrm{g}, g^{\prime}$ and $f_{k}$ are parameters, $\mathrm{H}$ is the usual Higgs doublet. $Y_{L}$ and $Y_{R}$ are hypercharge opreators have the following forms 


$$
\begin{aligned}
Y_{L} & =\left[\begin{array}{cc}
\frac{1}{3} \mathbf{1}_{3} & 0 \\
0 & -1
\end{array}\right], \\
Y_{R} & =\left[\begin{array}{cccc}
\frac{4}{3} \mathbf{1}_{3} & 0 & 0 & 0 \\
0 & -\frac{2}{3} \mathbf{1}_{3} & 0 & 0 \\
0 & 0 & -2 & 0 \\
0 & 0 & 0 & 0
\end{array}\right] .
\end{aligned}
$$

The field strength is defined through wedge product and derivative of gauge fields as follows

$$
F=D A+A \wedge A
$$

From Eq. (17) and (21), we can calculate the components of $\mathrm{F}$ as

$$
\begin{aligned}
F & =D X^{\mu} \wedge D X^{v} \frac{1}{2}\left(\partial_{\mu} A_{v}-\partial_{v} A_{\mu}+\left[A_{\mu}, A_{v}\right]\right) \\
& +2 D X^{\mu} \wedge D X^{5} \frac{1}{2}\left(\partial_{\mu}+\left(\tilde{A}_{\mu}-A_{\mu}\right)\right)\left(A_{5}+m\right) \\
& +D X^{5} \wedge D X^{5}\left(m\left(a_{5}+a_{5}^{\dagger}\right)+a_{5}^{+} a_{5}\right) \\
= & D X^{\mu} \wedge D X^{v} F_{\mu \nu}+2 D X^{\mu} \wedge D X^{5} F_{\mu 5} \\
& +D X^{5} \wedge D X^{5} F_{55}
\end{aligned}
$$

Let us calculate explicitly each component in terms of the physical boson and Higgs fields. The first component is

$$
\begin{aligned}
F_{\mu v}= & \frac{1}{2}\left(\partial_{\mu} A_{v}-\partial_{v} A_{\mu}+\left[A_{\mu}, A_{v}\right]\right) \\
= & f_{\mu \nu L}+f_{\mu \nu R} \\
= & \frac{1}{2}\left(\partial_{\mu} a_{v L}-\partial_{\nu} a_{\mu L}+\left[a_{\mu L}, a_{v L}\right]\right) \\
& +\frac{1}{2}\left(\partial_{\mu} a_{v R}-\partial_{\nu} a_{\mu R}+\left[a_{\mu R}, a_{v R}\right]\right) \\
= & \frac{g}{4} W_{\mu \nu} \otimes 1_{4}-g^{\prime} \frac{Y_{L} \otimes 1_{2}+Y_{R}}{4} B_{\mu \nu}
\end{aligned}
$$

where

$$
\begin{aligned}
W_{\mu v} & =\tau^{a}\left(\partial_{\mu} W_{v}^{a}-\partial_{v} W_{\mu}^{a}+\frac{g}{2} f_{a b c}\left[W_{\mu}^{b}, W_{v}^{c}\right]\right), \\
B_{\mu v} & =\partial_{\mu} B_{v}-\partial_{v} B_{\mu} .
\end{aligned}
$$

The second component is 


$$
F_{\mu 5}=\frac{1}{2}\left(\partial_{\mu}+\left(\tilde{A}_{\mu}-A_{\mu}\right)\right)\left(A_{5}+m\right)=f_{\mu 5 L}+f_{\mu 5 R}
$$

where $f_{\mu 5 L}, f_{\mu 5 R}$ is

$$
\begin{aligned}
& f_{\mu S L}=\frac{1}{2}\left(\partial_{\mu}+\left(a_{\mu R}-a_{\mu L}\right)\right)\left(a_{5}+m\right) \\
& =\frac{1}{2} f_{k}\left(\left[\begin{array}{cc}
\partial^{\mu} h_{0} & \partial^{\mu} h_{1}^{*} \\
-\partial^{\mu} h_{1} & \partial^{\mu} h_{0}^{*}
\end{array}\right]+\left[\begin{array}{cc}
h_{0} & h_{1}^{*} \\
-h_{1} & h_{0}^{*}
\end{array}\right]\left(-g W_{\mu}^{a}(x) \frac{\tau^{a}}{2} \otimes \mathbf{1}_{4}+g^{\prime} \frac{Y_{L} \otimes \mathbf{1}_{2}-Y_{R}}{2} B_{\mu}(x)\right)\right) \\
& f_{\mu S L}=\frac{1}{2}\left(\partial_{\mu}+\left(a_{\mu R}-a_{\mu L}\right)\right)\left(a_{5}+m\right) \\
& =\frac{1}{2} f_{k}\left(\left[\begin{array}{ll}
\partial^{\mu} h_{0} & \partial^{\mu} h_{1}^{*} \\
-\partial^{\mu} h_{1} & \partial^{\mu} h_{0}^{*}
\end{array}\right]-\left[\begin{array}{cc}
h_{0} & h_{1}^{*} \\
-h_{1} & h_{0}^{*}
\end{array}\right]\left(-g W_{\mu}^{a}(x) \frac{\tau^{a}}{2} \otimes \mathbf{1}_{4}+g^{\prime} \frac{Y_{L} \otimes \mathbf{1}_{2}-Y_{R}}{2} B_{\mu}(x)\right)\right)
\end{aligned}
$$

The third component is

$$
\begin{aligned}
F_{55} & =m\left(a_{5}^{\dagger}+a_{5}\right)+a_{5}^{\dagger} a_{5} \\
& =m\left(f_{k}\left(h_{0}^{*}+h_{0}\right)-2 m\right) \\
& +\left(f_{k} h_{0}^{*}-m\right)\left(f_{k} h_{0}-m\right)+f_{k}^{2} h_{1}^{*} h_{1} \\
& =f_{k}^{2}\left(\left(h_{0}^{*} h_{0}+h_{1}^{*} h_{1}\right)-\frac{m^{2}}{f_{k}^{2}}\right) \\
& =f_{k}^{2}\left(\bar{H} H-\frac{m^{2}}{f_{k}^{2}}\right) .
\end{aligned}
$$

The Lagrangian of the gauge sector now is calculated as

$$
\begin{aligned}
& \mathcal{L}_{g}=\frac{1}{f_{k}^{2}}\langle F, F\rangle \\
& =\frac{1}{f_{k}^{2}} \operatorname{Trace}\left(F_{\mu \nu}^{\dagger} F^{\mu \nu}+2 F_{\mu 5}^{\dagger} F^{\mu 5}+F_{55}^{\dagger} F^{55}\right) .
\end{aligned}
$$

Let calculate the first term in the Lagrangian, we have

$$
\begin{aligned}
& F_{\mu \nu}^{\dagger} F^{\mu \nu}=f_{\mu \nu L}^{\dagger} f_{L}^{\mu \nu}+f_{\mu \nu R}^{\dagger} f_{R}^{\mu \nu} \\
& =\frac{g^{2}}{16} W_{\mu \nu} W^{\mu v} \otimes 1_{4}+g^{\prime 2} \frac{Y_{L}^{2} \otimes 1_{2}+Y_{R}^{2}}{16} B_{\mu \nu} B^{\mu \nu}
\end{aligned}
$$

so we can get the trace of equation (35)

$$
\operatorname{Trace}\left(F_{\mu \nu}^{\dagger} F^{\mu v}\right)=\frac{g^{2}}{4} W_{\mu \nu} W^{\mu v}+g^{\prime 2} \frac{5}{6} B_{\mu \nu} B^{\mu v}
$$


The second term can be derive similarly

$$
\begin{gathered}
F_{\mu 5}^{\dagger} F^{\mu 5}=\left(f_{\mu 5 L}^{\dagger}+f_{\mu 5 R}^{\dagger}\right)\left(f^{\mu 5 L}+f^{\mu 5 R}\right) \\
=f_{\mu 5 L}^{\dagger} f^{\mu 5 L}+f_{\mu 5 R}^{\dagger} f^{\mu 5 R} \\
\operatorname{Trace}_{\mu 5}^{\dagger} F^{\mu 5}=\frac{1}{2} \operatorname{Trace}\left(f_{\mu 5 L}^{\dagger} f^{\mu 5 L}+f_{\mu 5 R}^{\dagger} f^{\mu 5 R}\right) \\
=\frac{1}{4} \operatorname{Trace}\left(\partial_{\mu} a_{5}^{\dagger} \partial^{\mu} a_{5}+\left(a_{5}^{\dagger}+m\right)\left(a_{5}+m\right)\left(a_{\mu R}-a_{\mu L}\right)\left(a_{R}^{\mu}-a_{L}^{\mu}\right)\right) \\
=\frac{1}{4} f_{k}^{2} \operatorname{Trace}\left[\begin{array}{cc}
\partial_{\mu} h_{0}^{*} \partial^{\mu} h_{0}+\partial_{\mu} h_{1}^{*} \partial^{\mu} h_{1} & 0 \\
0 & \partial_{\mu} h_{0}^{*} \partial^{\mu} h_{0}+\partial_{\mu} h_{1}^{*} \partial^{\mu} h_{1}
\end{array}\right] \\
+f_{k}^{2} \operatorname{Trace}\left(\left[\begin{array}{cc}
h_{0} h_{0}^{*}+h_{1} h_{1}^{*} & 0 \\
0 & h_{0} h_{0}^{*}+h_{1} h_{1}^{*}
\end{array}\right] \times\right. \\
\left.\left(g^{2} W_{\mu}^{a} W^{\mu b} \frac{\tau^{a} \tau^{b}}{4} \otimes \mathbf{1}_{4}+g^{\prime 2} \frac{Y_{L}^{2} \otimes \mathbf{1}_{2}+Y_{R}^{2}}{4} B_{\mu} B^{\mu}\right)\right) .
\end{gathered}
$$

So we have

$$
\operatorname{TraceF}_{\mu 5}^{\dagger} F^{\mu 5}=\frac{1}{2} f_{k}^{2} \nabla_{\mu} \bar{H} \nabla H .
$$

where the covariant derivative of the Higgs field define as

$$
\nabla_{\mu}=\partial_{\mu}+i\left(\frac{g}{2} A_{\mu}+\frac{g^{\prime}}{2} W_{\mu}^{i} \sigma^{i}\right)
$$

The last term can be calculated as follow

$$
\begin{gathered}
F_{55}^{\dagger} F^{55}=f_{k}^{4}\left(\bar{H} H-\frac{m^{2}}{f_{k}^{2}}\right)^{2}, \\
\text { Trace }_{55}^{\dagger} F^{55}=f_{k}^{2} V(\bar{H}, H) .
\end{gathered}
$$

Finally, replace equation (36), (38) and (41) into equation (34) we obtain

$$
\mathcal{L}_{g}=-\frac{1}{4}\left(F^{2}+G^{2}\right)+\frac{1}{2} \nabla_{\mu} \bar{H} \nabla^{\mu} H+V(\bar{H}, H) .
$$

In order to have the right factors for kinetic terms of the gauge fields, we imply that

$$
\begin{aligned}
& \frac{1}{f_{k}^{2}} \frac{g^{2}}{4}=\frac{1}{4} \rightarrow f_{k}=g \\
& \frac{1}{g^{2}} g^{\prime 2} \frac{5}{6}=\frac{1}{4} \rightarrow \mathrm{g}^{\prime}=\mathrm{g} \sqrt{\frac{3}{10}} .
\end{aligned}
$$

Hence the Weinberg angle is calculated explicitly as follows

$$
\sin ^{2} \theta_{W}=\frac{g^{\prime 2}}{g^{2}+g^{\prime 2}}=\frac{3}{13} \approx 0.23077
$$


The deviation of this prediction is just $0.1 \%$ compared to the experimental value.

\section{Comparison with the unified model based on the gauge symmetry $\mathrm{SU}(2 \mid 1)$}

In this section we discuss about six dimensonal Yang-Mill theory introduced by Fairlie [5, 6]. The gauge field $A_{m}, m=1, \ldots, 6$ has the form [5]

$$
\begin{aligned}
& A_{\mu}=\left(\begin{array}{cc}
g A_{\mu}-g^{\prime} B_{\mu} I & 0 \\
0 & \lambda g^{\prime} B_{\mu}
\end{array}\right), \\
& \mu=1, \ldots, 4 .
\end{aligned}
$$

Where $\mathrm{g}$ and $g^{\prime}$ are the usual coupling constants. In this framework, the gauge fields $A_{\mu}$ and $B_{\mu}$ transforms under $\mathrm{SU}(2)$ and $\mathrm{U}(1)$ respectively, where $\mathrm{I}$ is the $2 \times 2$ unit matrix, $\lambda$ is an arbitrary parameter. The components in extra directions are specified as

$$
\begin{aligned}
& A_{5}=g\left(\begin{array}{cc}
0 & i \phi \\
-i \phi^{+} & k
\end{array}\right), \\
& A_{6}=g\left(\begin{array}{cc}
0 & \phi \\
\phi^{+} & k
\end{array}\right) .
\end{aligned}
$$

Where $\phi$ is the Higgs doublet and $\mathrm{k}$ is an ad hoc parameter. The field strength $F_{m n}$ obtained by commutation of covariant derivative $[5,6]$

$$
\begin{aligned}
& F_{\mu \nu}=\frac{1}{g}\left(\begin{array}{cc}
g F_{\mu \nu}(A)-g^{\prime} F_{\mu \nu}(B) I & 0 \\
0 & \lambda g^{\prime} F_{\mu \nu}(B)
\end{array}\right), F_{\mu 5}=\left(\begin{array}{cc}
0 & i D_{\mu} \phi \\
-i\left(D_{\mu} \phi\right)^{+} & 0
\end{array}\right), \\
& F_{\mu 6}=\left(\begin{array}{cc}
0 & D_{\mu} \phi \\
\left(D_{\mu} \phi\right)^{+} & 0
\end{array}\right), F_{56}=2 g\left(\begin{array}{cc}
\phi \phi^{+} & (1-i) k \phi \\
(1+i) k \phi^{+} & -\phi^{+} \phi
\end{array}\right),
\end{aligned}
$$

where

$$
D_{\mu} \phi=\left(\partial_{\mu}+g A_{\mu}-g^{\prime}(1+\lambda) B_{\mu} I\right) \phi .
$$

The Lagrangian can be calculated as follows

$$
\begin{aligned}
-\frac{1}{4} F_{m n}^{2}= & -\frac{1}{4} F_{\mu \nu}^{2}(A) \\
& -\frac{1}{4}\left(g^{\prime} / g\right)^{2}\left(2+\lambda^{2}\right) F_{\mu \nu}^{2}(B) \\
& -\frac{1}{2}\left|D_{\mu} \phi\right|^{2}-g^{2}\left(\phi^{+} \phi\right)^{2}-2 k^{2} g^{2} \phi^{+} \phi .
\end{aligned}
$$

The Weinberg angle is specified as follows 


$$
\tan \theta_{w}=g^{\prime} / g=\left(1+\frac{1}{2} \lambda^{2}\right)^{-1 / 2}
$$

We have two alternatives for the right kinetic terms of the gauge fields in this theory with $\lambda= \pm 2$ leading to the same value of $\tan \theta_{w}$.Therefore, we obtain

$$
\begin{aligned}
& g^{\prime} / g=1 / \sqrt{3} \\
& \rightarrow \sin ^{2} \theta_{W}=\frac{g^{\prime 2}}{g^{2}+g^{\prime 2}}=\frac{1}{4}=0.25 .
\end{aligned}
$$

This value of is significantly greater than the experiment value. Therefore, this theory will be effective at an energy much higher than the electroweak one with the assumption that no new physics emerges in the $\mathrm{TeV}$ range.

\section{Conclusions}

The geometric approach discussed in this paper is based on the DKKT, with the internal space having only two points. It is straightforward to generalize into $\mathrm{N}$ points and arbitray number of the internal dimensions. DKKT is a good way to unify all the interactions and Higgs field without resorting to infinite tower of massive modes following Einstein's General Relativity. Surprisingly, the geometric approach give the result with excellence agreement with the experiments at the electroweak energy scale. So DKKT is valid at the currently accessible energy (unlike other unified theories). In the geometric approach, we don't need to assume higher gauge symmetries to predict the Weinberg angle. More phenomenological predictions of DKKT are in progress.

\section{Acknowledgement}

Thanks are due to Nguyen Ai Viet, Tran Minh Hieu and Pham Tien Du for their collaborations and discussions. The research is funded by Vietnam National Foundation for Science and Technology Development (NAFOSTED) under grant number 103.01-2017.319.

\section{References}

[1] T.P. Cheng, L.F. Li., Gauge theory of elementary particle physics, Clarendon Press, Oxford, 1984.

[2] L.B. Okun, Leptons and quarks, Elsevier, North-Holland, 2013.

[3] T.D. Lee, Particle physics and introduction to field theory, Contemporary Concepts in Physics Vol. 1, Harwood Academic, New York, 1981.

[4] C. Amsler, Review of Particle Physics - Electroweak model and constraints on new physics, Physics Letters (2008) B-667.

[5] D. Fairlie, Two consistent calculations of the Weinberg angle, Journal of Physics G: Nuclear Physics 5 (1979) L55.

[6] D. Fairlie, Higgs fields and the determination of the Weinberg angle, Physics Letters B 82 (1979) 97-100.

[7] G. Landi, N.A. Viet, K.C. Wali, Gravity and electromagnetism in noncommutative geometry, Physics Letters B 326 (1-2) (1994) 45-50.

[8] N.A. Viet, K.C. Wali, A discretized version of Kaluza-Klein theory with torsion and massive fields, International Journal of Modern Physics A 11 (13) (1996) 2403-2418. 
[9] N.A. Viet, K.C. Wali, Noncommutative geometry and a discretized version of Kaluza-Klein theory with a finite field content, International Journal of Modern Physics A. 11(3) (1996): 533-551.

[10] N.A. Viet, K.C. Wali, Chiral spinors and gauge fields in noncommutative curved space-time, Physical Review D, 67(12) (2003) 124029.

[11] A.V. Nguyen, T.D. Pham, Non-Abelian gauge fields as components of gravity in the discretized Kaluza-Klein theory. Modern Physics Letters A. 32(18) (2017) 1750095.

[12] N.A. Viet, N.V. Dat, N.S. Han, K.C. Wali, Einstein-Yang-Mills-Dirac systems from the discretized Kaluza-Klein theory. Physical Review D. 95(3) (2017) 035030.

[13] A.H. Chamseddine, G. Felder, J. Fröhlich, Gravity in non-commutative geometry. Communications in Mathematical Physics 155(1) (1993) 205-217.

[14] A. Connes and J. Lott, Particle models and noncommutative geometry. Nucl. Phys. B, 18 (1991) 29-47.

[15] T. Kaluza, Zum unitätsproblem der physik, Sitzungsber. Preuss. Akad. Wiss. Berlin (Math. Phys.) (1921) (arXiv: 1803.08616) 966-972.

[16] O. Klein, Quantum theory and five-dimensional theory of relativity, 1926 Z. Phys. 37 (1987) 895. 